\title{
PÊNFIGO VULGAR \\ - a importância do conhecimento do cirurgião dentista para um correto diagnóstico•
}

\begin{abstract}
Deyvid Silva Rebouças*, Lucas Souza Cerqueira*, Tila Fortuna Costa*, Thaise Gomes Ferreira*, Roberta Catapano Naves**, Livia Prates Soares Zerbinati***

Autor correspondente: Deyvid da Silva Rebouças. Fazenda grande 1, Quadra C, Bloco 05, Apartamento 104. Cajazeiras. Salvador - Ba. CEP: 41340-100. E-mail: dsr.ctbmf@gmail.com. Tel: (71) 9178-3132.

* Residente Cirurgia e Traumatologia Buco-Maxilo-Facial Hospital Geral Roberto Santos/Escola Bahiana de Medicina e Saúde Pública

** Mestre em Clinica Odontológica. Professora de Periodontia da pós-graduação da ABO-BA e da EBMSP

*** Doutora em Cirurgia e Traumatologia Buco-Maxilo-Facial. Preceptora do Serviço de Cirurgia e Traumatologia Buco-Maxilo-Facial/ EBMSP
\end{abstract}

\section{Resumo}

Pênfigo é a denominação geral de um grupo de doenças autoimunes raras que acometem pele e mucosas e apresenta-se com formação de bolhas intraepidérmicas. O Pênfigo Vulgar (PV) é uma doença vésico-bolhosa crônica, rara, de natureza autoimune, quando não diagnosticada e tratada na sua fase inicial apresenta prognóstico grave. Este trabalho tem como objetivo relatar um caso clínico de uma paciente do gênero feminino, 32 anos de idade que apresentava manifestação de Pênfigo Vulgar. Asmanifestações bucais são, em sua maioria, os primeiros sinais da doença e o cirurgião-dentista possui papel fundamental no diagnóstico precoce.

Palavras-chave: Autoimunidade; Dermatopatia, Vesiculobolhosa; Pênfigo.

\section{PEMPHIGUS VULGARIS \\ - the importance of knowledge of the surgeon dentist for a correct diagnosis}

\begin{abstract}
Pemphigus is the general name for a group of rare autoimmune diseases that affect the skin and mucous membranes and is presented with the formation of intraepidermal blisters. Pemphigus vulgaris (PV) is a chronic rare vesicular-bullous autoimmune disease that when not diagnosed and treated in its early
\end{abstract}


stages has severe prognosis. This study aims to report a clinical case of a female patient, 32 years of age who had manifestation of pemphigus vulgaris. The oral manifestations are, in most cases, the first signs of the disease and the dentist has a primary role in the early diagnosis.

Keywords: Autoimmunity; Skin diseases, Vesiculobullous; Pemphigus.

\section{INTRODUÇÃOO}

Pênfigo é a denominação geral de um grupo de doenças autoimunes raras que acomete pele e mucosas, caracterizada pela presença de auto-anticorpos que atuam nas proteínas desmossômicas encontradas nas junções epiteliais dos tecidos de revestimento. (1) Há quatro tipos principais de pênfigo: foliáceo, eritematoso, vulgar e vegetante.(2) O Pênfigo Vulgar, $(\mathrm{PV})$, é o tipo de pênfigo mais comum e corresponde a aproximadamente $70 \%$ dos casos. ${ }^{(1)}$

As manifestações iniciais do pênfigo vulgar frequentemente envolvem a mucosa oral antes do aparecimento de lesões cutâneas. ${ }^{(2,3)}$ As bolhas presentes na mucosa bucal são efêmeras, rompem-se rapidamente, deixam áreas ulceradas de tamanho e formas variadas, dificultam as funções mastigatórias, de deglutição e fonação do paciente, (4) porém raramente os pacientes percebem a formação de vesículas ou bolhas intraorais, sendo também rara a observação desses sinais pelos cirurgiões-dentistas. ${ }^{(2)}$

O caráter imunológico do PV está estabelecido na literatura científica, entretanto a etiologia da doença permanece desconhecida. Frequentemente, o seu reconhecimento é somente após o aparecimento das lesões cutâneas vesículo-bolhosas, o que retarda seu diagnóstico e tratamento. ${ }^{(5)}$

Alguns fatores têm sido discutidos como prováveis desencadeadores da doença, tais como medicamentos, procedimentos cirúrgicos, queimadura térmica, radiação ultravioleta, radioterapia, estresse emocional e consumo de alguns alimentos..$^{(2,6)}$

Embora possa ser identificada em crianças e idosos, a média de idade de aparecimento das lesões do PV é entre quarenta e sessenta anos e não apresenta maior incidência entre homens e mulheres. ${ }^{(2,7)}$

O sinal de Nikolsky é uma manobra auxiliar no diagnóstico do $P V$, entretanto, não é patognomônico. ${ }^{(8)}$ A biópsia é um elemento importante no diagnóstico e deve ser realizada, se possível, numa bolha intacta. ${ }^{(9)}$ Clinicamente, as lesões bucais do Pênfigo Vulgar devem ser diferenciadas das infecções virais e do eritema multiforme que são condições de natureza aguda. $(2,10)$

As opções de tratamento e seus efeitos colaterais influenciam no cotidiano dos pacientes. $O$ tratamento é essencialmente sintomático e consiste basicamente no uso de corticóides sistêmico e em alguns casos associa-se a imunossupressores, além de antibióticos e antifúngicos para prevenção de infecções secundárias. ${ }^{(8,11)}$ A terapia farmacológica controla, entretanto, não cura a doença, sendo necessário o acompanhamento destes pacientes por longos períodos. ${ }^{(3)}$

O presente trabalho tem como objetivo reportar um caso de diagnóstico e controle de pênfigo vulgar em um paciente que compareceu a um serviço de Cirurgia e Traumatologia Buco-Maxilo-Facial, além de discutir as características clínicas encontradas, 
enfatizando a importância do cirurgião-dentista no diagnóstico dessa doença autoimune.

\section{RELATO DE CASO}

Paciente de gênero feminino, 32 anos, compareceu ao ambulatório de Cirurgia e Traumatologia Buco-Maxilo-Facial, do Hospital Geral Roberto Santos (HGRS), em Salvador (BA), com relato de aparecimento de múltiplas lesões ulceradas em boca e em lábio inferior com sintomatologia dolorosa há 3 meses (Figuras 1 e 2).

O exame físico revelou inúmeras ulcerações em mucosa oral (mucosa jugal, labial e gengiva) e em tecido cutâneo do corpo (Figura 3) . Foi realizado pressão manual em um ponto da mucosa oral para indentificar sinal de Nikolsky e parte da superfície epitelial destacou-se, o que sugere o Pênfigo Vulgar como suspeita diagnóstica. A conduta inicial estabelecida foi realização de biopsia incisional (Figura 4) e prescrição de Predinisona 4omg/dia, inicialmente para controle das manifestações agudas da doença e aplicação tópica de lidocaína 10\% spray na mucosa antes da alimentação para minimizar o desconforto da paciente.

Após 07 dias da corticoterapia, houve cicatrização de quase todas as úlceras em cavidade bucal e melhora do desconforto da paciente. Iniciou-se o desmame da corticoterapia para diminuição da dose diária do corticóide. O exame histopatológico apresentou uma fenda entre o tecido epitelial e o conjuntivo com infiltrado brando composto por células inflamatórias crônicas sugestivo de pênfigo vulgar. A paciente foi encaminhada para o dermatologista para acompanhamento e controle da lesões em pele e a mesma continua em acompanhamento no serviço de Cirurgia e Traumatologia Buco-Maxilo-Facial do HGRS.

A paciente encontra-se com a doença controla$\mathrm{da}$, sem queixas, uso de $2,5 \mathrm{mg} /$ dia de predinisona para estabilização do PV e acompanhamento multiprofissional.

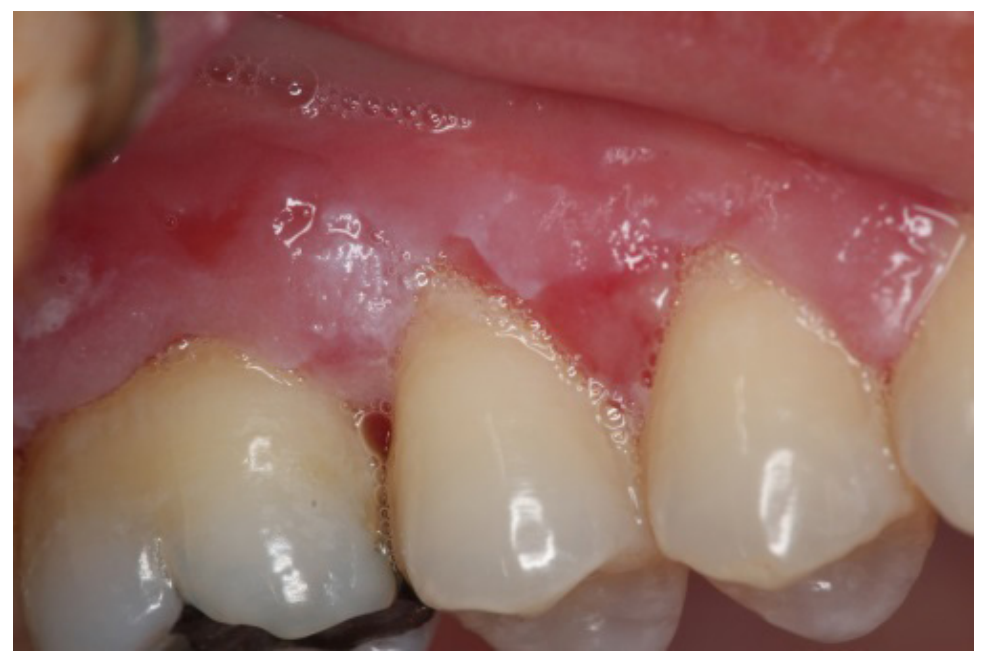

Figura 1 - Imagem intra-oral, mostrando o aspecto clínico da gengiva da paciente e evidenciando áreas com descamação da mucosa 


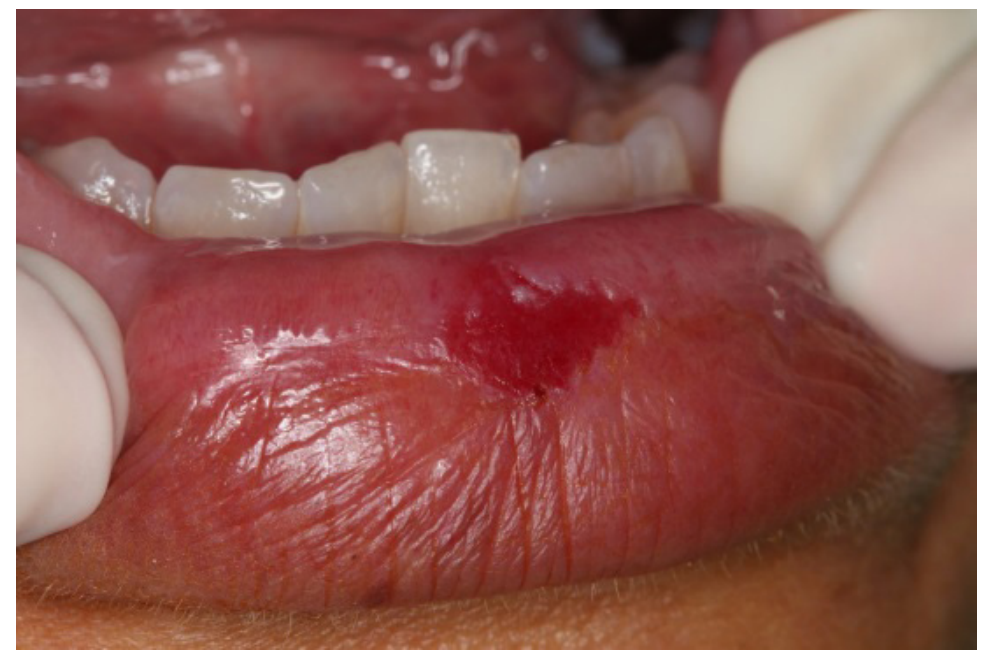

Figura 2 - Imagem do lábio inferior, evidenciando a presença de lesão ulcerativa nessa região

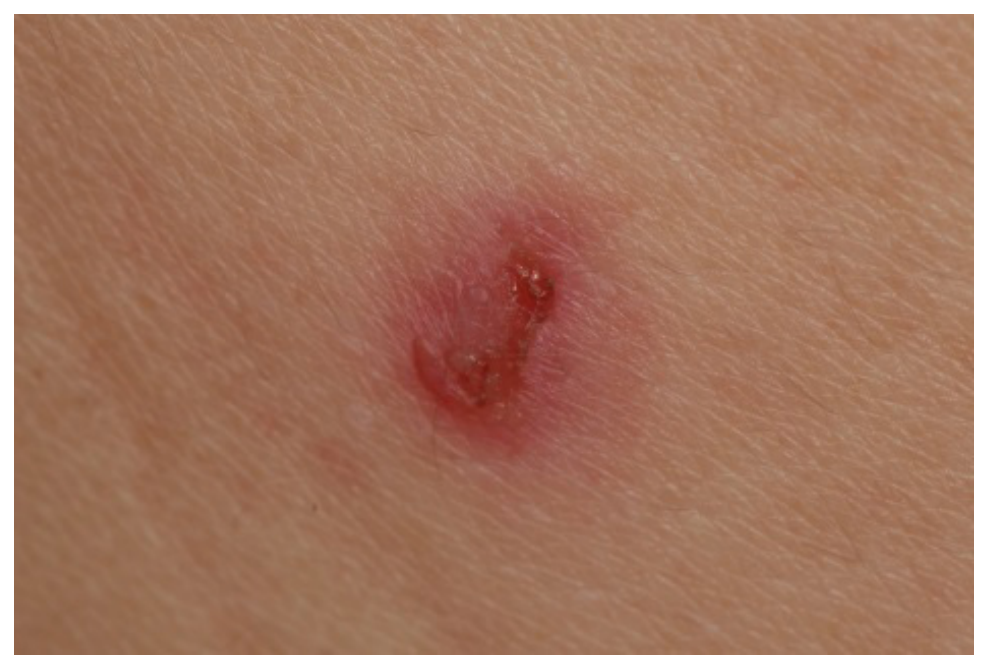

Figura 3 - Úlcera localizada em tecido cutâneo do corpo da paciente

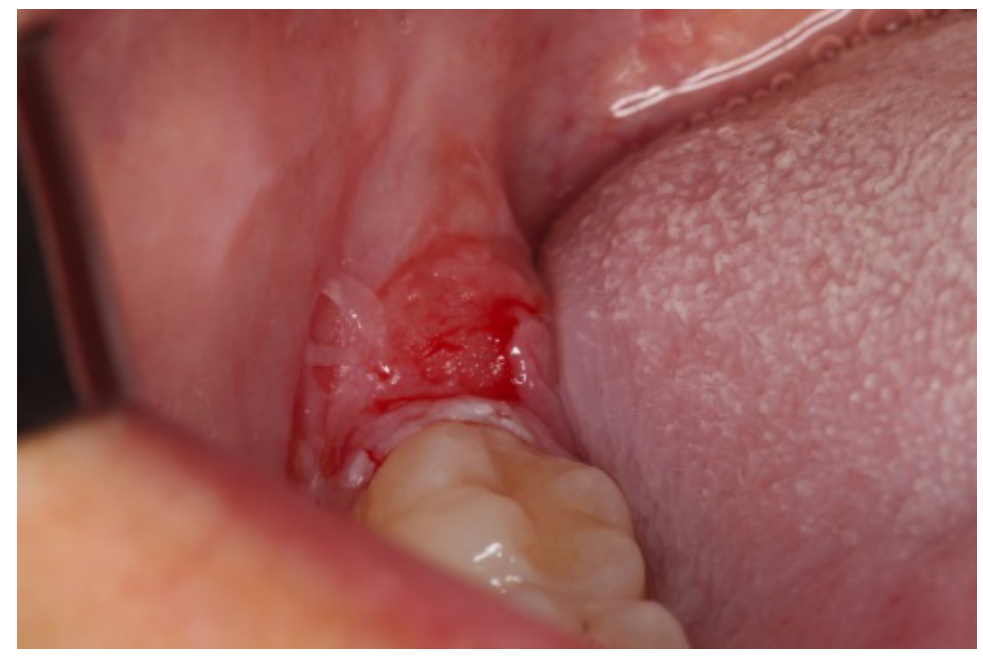

Figura 4 - Aspecto pós-operatório, mostrando a área da lesão após realização de biópsia incisional 


\section{DISCUSSÃO}

As doenças autoimunes apresentam aumento da incidência devido ao envelhecimento populacional. ${ }^{(13)}$ Os idosos são mais suscetíveisàs doenças autoimunes bolhosas. ${ }^{(14)}$ Pênfigo é a denominação geral de um grupo de doenças autoimunes raras que acomete pele e mucosas, caracterizadas pela formação de bolhas intraepidérmicas, como produto da acantólise. ${ }^{(15)}$

O pênfigo pode ser encontrado em várias faixas etárias, apesar de ser um evento incomum na infância. Os casos geralmente são observados em adultos entre quarenta e sessenta anos e não apresentam maior ocorrência entre os gêneros. ${ }^{(16,17)}$ No entanto, o caso relatado apresenta lesões de pênfigo em indivíduo adulto jovem.

As lesões tem início geralmente insidioso e são disfarçadas através de ulcerações que cicatrizam em algumas semanas. O Pênfigo vulgar frequentemente acomete a mucosa oral antes do aparecimento de lesões cutâneas, com predileção pelo palato, mucosa labial, mucosa jugal, ventre da língua e gengiva. As lesões bucais podem manifestar-se até dois anos antes da eclosão sistêmica da doença. ${ }^{15,16)}$ As lesões ulcerativas do PV acometeram mucosa jugal, labial, gengiva e precedeu as manifestações cutâneas, conforme a literatura supracitada.

A mucosa oral é freqüentemente afetada por doenças autoimunes bolhosas que precedem as lesões cutâneas e podem ser os únicos sinais presentes da doença. As gengivites descamativas são causadas por várias patologias mucocutâneas, sendo mais frequentes nos penfigóides e líquen plano erosivo e menos frequente no PV. ${ }^{(18)}$ No presente caso as gengivites descamativas foram identificadas, porém outros sinais conduziram para a suspeita diagnóstica de pênfigo vulgar.

Doenças erosivas ou ulcerativas afetam a mucosa oral ou gengiva e podem apresentar um difícil diagnóstico clinico. As lesões bucais do PV devem ser diferenciadas das infecções virais e do eritema multiforme que são condições de natureza aguda. ${ }^{(19)}$
O diagnóstico precoce é essencial para o controle do curso e o prognóstico de uma dermatose bolhosa autoimune, o que pode retardar ou mesmo inibir a disseminaçãodas lesões observadas. ${ }^{(14,19)}$

O sinal de Nikolsky constitui uma manobra semiológica que pode ser utilizada como critério de diagnóstico de pênfigo vulgar, porém, não é um achado patognomônico. $A$ ausência de padronização da execução da manobra (uso de clipes, dedo, espátula de madeira, entre outros) e a dor que este provoca, tornam seu uso limitado. $O$ sinal é obtido através de friç̧ão ou pressão em uma área de pele ou mucosa, aparentemente normal, que pode provocar uma reação de bolha hemorrágica ${ }^{(15)}$ ou desprendimento de tecido. ${ }^{(20)}$ Esta manobra foi fundamental para nortear a suspeita diagnóstica do caso em estudo.

O diagnóstico é firmado através do exame histopatológico. ${ }^{(3)}$ De acordo com Santos et al. 2009, a imunofluorescência deve ser solicitada para os casos que o exame histopatológico não seja elucidativo. A biópsia é um elemento diagnóstico importante e deve ser realizada, se possível, em uma bolha intacta. ${ }^{(3)}$ No caso apresentado, o diagnóstico foi estabelecido pelo exame clínico e confirmado pelo histopatológico através de biópsia incisional.

Os índices elevados de morbidade e mortalidade previamente associados ao pênfigo vulgar, representavam aproximadamente $80 \%$, atualmente com a terapia imunossupressora, houve redução acentuada desse índice. ${ }^{(2,17)} \mathrm{O}$ tratamento inicial com corticosteróides sistêmicos, como prednisona, induz a remissão das úlceras e deve-se manter doses mínimas por um período de estabilização da doença. ${ }^{(12)}$ Conseguiu-se controlar as manifestações clínicas da doença com o uso de prednisona conforme a literatura.

\section{CONSIDERAÇÕES FINAIS}

O sinal de Nikolsky possibilita ao profissional direcionar as suspeitas diagnósticas a um grupo de 
doenças que desprendem epitélio. O principal valor deste exame é diferenciar as bolhas provocadas por processos autoimunes daquelas que são desencadeadas por traumas.

O diagnóstico e o tratamento de desordens imunologicamente mediadas, como o Pênfigo Vulgar, nos estágios iniciais da doença permitem obter o controle desta sem graves consequências para o paciente. Então, torna-se de fundamental importância que o cirurgião-dentista conheça os sinais e sintomas e estabeleça o diagnóstico precoce, visto que essas lesões geralmente acometem inicialmente a região maxilofacial.

\section{REFERÊNCIAS}

1. Dabelsteen E. Molecular biological aspects of acquired bullous diseases. Crit Rev Oral Biol Med 1998;9(2):162-178.

2. Neville BW, Damm DD, Allen CM, Bouquot JE. Patologia oral \& maxilofacial. Rio de Janeiro: Guanabara-Koogan; 2004. p. 617-75.

3. Miziara, ID, Ximenes Filho JA, Ribeiro FC, Brandão AL. Acometimento oral no pênfigo vulgar. Rev. bras. otorrinolaringol. 2003;69(3):327-31.

4. Regezi JA, Sciubba JJ. Patologia bucal: correlações clinicopatológicas. Rio de Janeiro: Guanabara-Koogan; 2000. p. 1-26.

5. Silva $A V$, Valones MA, Guimarães RP, Castro JF, Carvalho AA. A therapeuticoption for diseasecontrol. Gen Dent. 2008;56:700-03.

6. Cohen SS, Weinstein MD, Herndier BG, Anhalt GJ, Blauvelt $A$. No evidence of human herpesvirus 8 infection in patients with paraneoplastic pemphigus, pemphigus vulgaris, or pemphigus foliaceus. J Invest Dermatol. 1998;111:781-83.

7. Bickle KM, Roark TR, Hsu S. Autoimmune bullous dermatoses: a review. Am Fam Physician. 2002;65(9):1861-70.

8. Tommasi AF, Sugaya NN. Lesões ulcerativas e vesicobolhosas. In: Tommasi AF. Diagnóstico em patologia bucal. São Paulo: Editora Pancast; 2002. p. 139-57.
9. Carranza AF, Newman MG, Henry HT, Klokkevold PR. Periodontia clínica. In: Shklar G. Gengivite descamativa e doenças da mucosa oral. Rio de Janeiro: Guanabara-koogan;1992, p. 142-45.

10. Greenberg MS. Ulcerative, Vesicular, and Bullous Lesions. In: Glick M, Greenberg MS, Ship JA. Burket's Oral Medicine. Hamilton: BC Decker; 2008. p. 61-65.

11. Robinson JC, Lozada-Nur F, Frieden I. Oral pemphigus vulgaris: a review of the literature in a report of the management of 12 cases. Oral Surg Oral Med Oral Pathol Oral Radio Endod. 1997;84(4):349-55.

12. Mutasim DF. Management of autoimmune bullous diseases: pharmacology and therapeutics. J Am Acad Dermatol 2004;51:859-77.

13. Schmidt E, Zilikens D. Diagnosis and Treatment of Patients with Autoimmune Bullous Disorders in Germany. Dermatol Clin. 2011;(29):663-671.

14. Mutasim DF. Autoimmune Bullous Dermatoses in the Elderly An Update on Pathophysiology, Diagnosis and Management. Drugs Aging. 2010;27(1):1-19.

15. Santos TS, Piva MR, Kumar PN, Martins Filho PRS, Reinheimer DM, Acevedo CR. Importância do cirurgião-dentista no diagnóstico precoce do pênfigo vulgar. RGO, 2009;57(3):351-355.

16. Sciubba JJ. Autoimmune Oral Mucosal Diseases: Clinical, Etiologic, Diagnostic, and Treatment Considerations. Dent Clin North Am. 2011;(55):89-103.

17. Santos TS, Lima FTB, Andrade ESS, Silva EDO, Gomes ACA. A Importância do diagnóstico precoce do penfigóide cicatricial: Relato de caso. Odontol. clin.-cient. 2010;9(1):83-87.

18. Ohta M, Osawa S, Endo H, Kuyama K, Yamamoto $\mathrm{H}$, Ito T. Pemphigus Vulgaris Confined to the Gingiva: A Case Report. Int J Dent. 2011.

19. Arisawa EAL, Almeida JD, Carvalho YR, Cabral LAG. Clinicopathological analysis of oral mucous autoimmune disease: A 27-year study. Med. oral patol. oral cir. bucal. 2008;13(2):E94-7.

2O. Moleri AB, Jordão MB, Ribeiro DMC, Moreira LC. Lesões orais do pênfigo vulgar: a importância do diagnóstico precoce. Acta Scientiae Medica Online. 2008;1(2): 72-9. 


\section{ANEXO I}

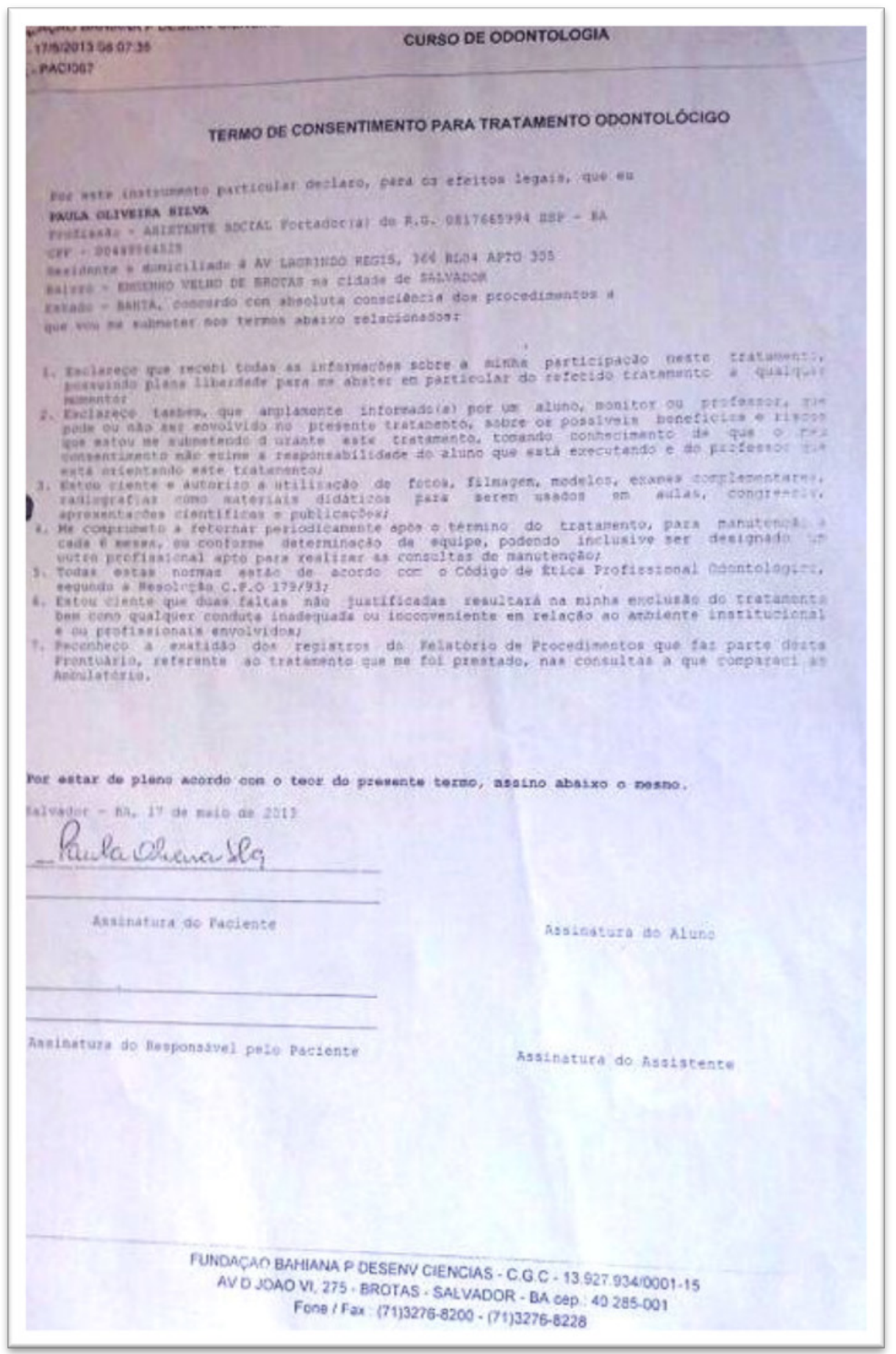

\section{DECLARAÇÃO DE RESPONSABILIDADE}

Certifico que participei da concepção do trabalho para tornar pública minha responsabilidade pelo seu conteúdo, não omitindo quaisquer ligações ou acordos de financiamento entre os autores e companhias que possam ter interesse na publicação deste artigo; - Certifico que o manuscrito é original e que o trabaIho, em parte ou na íntegra, ou qualquer outro trabalho com conteúdo substancialmente similar, de 
minha autoria, não foi enviado a outra Revista e não o será, enquanto sua publicação estiver sendo considerada pela revista bahiana de Odontologia, quer seja no formato impresso ou no eletrônico.

\section{PÊNFIGO VULGAR: A importância} do conhecimento do cirurgião dentista para um correto diagnóstico.

05 de dezembro de 2014

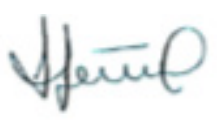

Tila Fortuna

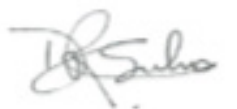

Deyvid Silva Rebouças

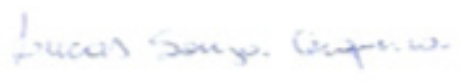

Lucas Souza Cerqueira

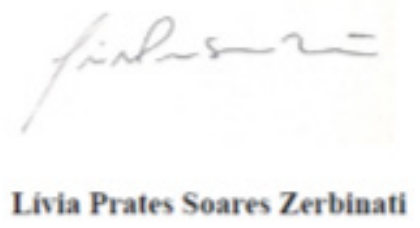

PÊNFIGO VULGAR: A importância do conhecimento do cirurgião dentista para um correto diagnóstico.

05 de dezembro de 2014
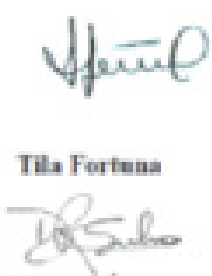

Deyid Silva Reboucas

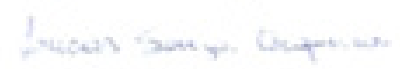

Lucm Seana Cerquarira

Livia Prates Searrs Zerbiaati

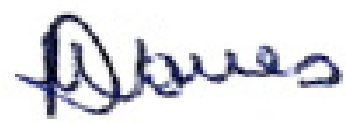

Roberta Catapano Noves

\section{Transferência de Direitos Autorais}

Declaro que, em caso de aceitação do artigo, a revista bahiana de Odontologia passa a ter os direitos autorais a ele referentes, que se tornarão propriedade exclusiva da Revista, vedado a qualquer reprodução, total ou parcial, em qualquer outra parte ou meio de divulgação, impressa ou eletrônica, sem que a prévia e necessária autorização seja solicitada e, se obtida, farei constar o competente agradecimento à Revista. 